\title{
HEIGHT OF OPERANDS OVER MONOIDS SATISFYING THE D.C.C. ON ORBITS
}

\author{
WILLIAM R. NICO
}

\begin{abstract}
The height, $h(X)$, of an operand $X$ over a monoid $S$ satisfying the d.c.c. on orbits is defined, and is compared to the saturation length, $\operatorname{sl}(X)$, of $X$. If $h(X)$ is finite, then $\operatorname{sl}(X) \leq h(X)$. If $X$ is saturated, then $h(X)$ must be a limit ordinal. If $h(X)$ is infinite, then it may be that $h(X)<\operatorname{sl}(X)$.
\end{abstract}

Let $S$ be a monoid, and let $X$ be a left $S$-operand. The identity of $S$ will always act as the identity transformation on $X$, and it may be that $X$ is empty. An operand $X$ is a generalized orbit if for every $x, y \in X$ there is a $z \in X$ such that $x, y \in S z$. Every operand is the union of its maximal generalized orbits (see [2]), and one calls an operand saturated if every maximal generalized orbit in the operand is contained in the union of the other maximal generalized orbit. If $X$ can be expressed as the union of a set of maximal generalized orbits, each of which is not contained in the union of the others, then $X$ admits an irredundant cover by maximal generalized orbits. Such a cover, if it exists, is unique. (See [2, Proposition 1.2].)

In [2] a descending chain of suboperands for an operand $X$ was constructed as follows.

Let $X_{0}=X$. If $X_{\sigma}$ has been defined for an ordinal $\sigma$, let

$$
M_{\sigma}=\left\{M \subset X_{\sigma}: M \text { is a maximal generalized orbit of } X_{\sigma}\right\},
$$

and set

$$
X_{\sigma+1}=\left\{x \in X_{\sigma}: x \in M \cap M^{\prime} \text { with } M, M^{\prime} \in \mathbb{M}_{\sigma} \text { and } M \neq M^{\prime}\right\} .
$$

If $\tau$ is a limit ordinal, then set

$$
X_{\tau}=\bigcap\left\{X_{\sigma}: \sigma<\tau\right\} .
$$

Received by the editors October 5, 1972 and, in revised form, April 29, 1974. AMS (MOS) subject classifications (1970). Primary 20M20.

Key words and phrases. Monoid, operand, semigroup, monoid acting on a set, transformation monoid. 
Then there is a least ordinal a such that $X_{a}=X_{a+1}$. Call this $a$ the saturation length of $X$, and denote it by $s l(X)$. If $X_{a} \neq \varnothing$, then it is known [2, Proposition 2.1] that $X_{\alpha}$ is saturated.

If $X$ is a left $S$-operand, let us say that two elements $x, y \in X$ are separated in $X$ if $x$ and $y$ are not contained in a common orbit in $X$.

Proposition 1. Let $X$ be a left S-operand.

(i) If $x \in X$ and if $M$ is a generalized orbit of $X$ such that $x \in M$, then $M=\bigcup\{S y: y \in M$ and $x \in S y\}$.

(ii) $x \in X$ lies in two distinct maximal generalized orbits of $X$ if and only if $x \in S y \cap S z$ with $y, z$ separated in $X$.

(iii) If $X_{\sigma}$ is as above, then $x \in X_{\sigma+1}$ if and only if there exist $y, z \epsilon$ $X_{\sigma}$ with $y, z$ separated in $X_{\sigma}$ and $x \in S y \cap S z$.

(iv) $X$ is saturated if and only if for all $x \in X$ there exist $y, z \in X$ with $y, z$ separated in $X$ and $x \in S y \cap S z$.

Proof. (i) For every $m \in M$ there exists $y_{m} \in M$ such that $x, m \in S y_{m}$. Hence $M \subset \bigcup\{S y: y \in M$ and $x \in S y\} \subset M$.

(ii) If such $y$ and $z$ exist, then they must lie in distinct maximal generalized orbits of $X$; hence $x$ lies in two distinct maximal generalized orbits of $X$.

Conversely, if no such $y, z$ exist, then $M=\bigcup\{S y: x \in S y\}$ is a generalized orbit of $X$. If $M^{\prime}$ is a generalized orbit of $X$ such that $x \in M^{\prime}$, then by (i) $M^{\prime} \subset M$, so that $M$ is the unique maximal generalized orbit of $X$ which contains $x$.

(iii) By definition, $x \in X_{\sigma+1}$ if and only if $x$ lies in two distinct maximal generalized orbits of $X_{\sigma}$. Thus (ii) applied to $X_{\sigma}$ gives (iii).

(iv) From [2, Proposition 1.3] it follows that $X$ is saturated if and only if $X=X_{1}$. Thus (iv) follows from (iii).

Say that a monoid $S$ satisfies the descending [ascending] chain condition on orbits if every left $S$-operand satisfies the d.c.c. [a.c.c.] on orbits. It is shown in [2] that the two chain conditions are independent, and that if $S$ satisfies the a.c.c. on orbits, then $S$ has no saturated operands.

The descending series defined above studies the way in which the orbits of an operand fit together. If the monoid $S$ satisfies the d.c.c. on orbits, then it is possible to define an ascending series of suboperands which gives further information about the way in which the orbits of the operand fit together. 
If $X$ is a left $S$-operand, and if $Y \subset X$ is a suboperand, call an orbit $U \subset X$, with $U \not \subset Y, Y$-distinguished if for every other orbit $V \subset X$ either $U \cap V \subset Y, U \subset V$, or $V \subset U$.

Proposition 2. If $S$ is a monoid satisfying the d.c.c. on orbits, then every $S$-operand $X$ has $Y$-distinguished orbits for every proper suboperand $Y \varsubsetneqq X$.

Proof. Since $S$ satisfies the d.c.c. on orbits, the collection of orbits $\{U: U$ is an orbit of $X$ and $U \not \subset Y\}$ has minimal elements. Any minimal element of this collection is a $Y$-distinguished orbit.

If $S$ satisfies the d.c.c. on orbits and if $X$ is a left $S$-operand, define an ascending chain of suboperands of $X$ as follows. Take $X^{0}=\varnothing$. Having defined $X^{\sigma}$ for an ordinal $\sigma$, if $X^{\sigma} \neq X$, set $X^{\sigma+1}=\bigcup\left\{U: U\right.$ is an $X^{\sigma}$. distinguished orbit of $X\} \cup X^{\sigma}$. If $\tau$ is a limit ordinal, set $X^{\tau}=\bigcup\left\{X^{\sigma}\right.$ : $\sigma<\tau\}$.

Proposition 3. Let $S$ be a monoid satisfying the d.c.c. on orbits and let $X$ be a left $S$-operand. If $X^{\sigma} \neq X$, then $X^{\sigma} \varsubsetneqq X^{\sigma+1}$. Hence there is an ordinal $\beta$ such that $X^{\beta}=X$.

Proof. If $X^{\sigma} \neq X$, then $X$ has $X^{\sigma}$-distinguished orbits, hence $X^{\sigma} \neq$ $X^{\sigma+1}$.

The existence of such a $\beta$ follows from cardinality considerations, and in fact $\operatorname{card}(\beta) \leq \operatorname{card}(X)$.

If $S$ satisfies the d.c.c. on orbits and if $X$ is a left $S$-operand, call the the least ordinal $\beta$ such that $X^{\beta}=X$ the height of $X$, and denote it by $h(X)$.

Theorem 4. Let $S$ be a monoid satisfying the d.c.c. on orbits, and let $X$ be a left $S$-operand.

(i) If some maximal generalized orbit of $X$ lies in the union of other maximal generalized orbits of $X$, then $h(X)$ must be infinite.

(ii) If $X$ is saturated, then $h(X)$ must be a limit ordinal.

Proof. (i) Let $M$ be a maximal generalized orbit of $X$ which lies in a union of other maximal generalized orbits. Then $M$ must be proper, i.e., not itself an orbit, so that for every $x \in M$ there is $y \in M$ such that $S x \varsubsetneqq$ Sy. We show by induction that $M \not \subset X^{n}$ for any integer $n$. Let $x_{1} \in M$. 
Since $M$ is contained in a union of other maximal generalized orbits, there is a $z_{1} \notin M$ with $x_{1} \in S z_{1}$. Since $M$ is proper, there is a $y_{1} \in M$ with $y_{1} \notin S z_{1}$ such that $x_{1} \in S y_{1}$. Thus $x_{1} \in S y_{1} \cap S z_{1}$, and $S y_{1}$ and $S z_{1}$ are incomparable, so that $y_{1} \notin X^{1}$. Hence $M \not \subset X^{1}$.

Suppose now that $M \not \subset X^{n}$. Let $x_{n+1} \in M$ with $x_{n+1} \notin X^{n}$. Then as above one finds $y_{n+1} \in M$ and $z_{n+1} \notin M$ with $y_{n+1}, z_{n+1} \notin X^{n}, S y_{n+1}$ and $S z_{n+1}$ incomparable, and $x_{n+1} \in S y_{n+1} \cap S z_{n+1}$. Hence, $y_{n+1} \notin X^{n+1}$ so that $M \not \subset X^{n+1}$. Therefore by induction, $M \not \subset X^{n}$ for any integer $n$, so that $h(X)$ must be infinite.

(ii) It suffices to show that if $X^{\sigma} \neq X$ for some ordinal $\sigma$, then $X^{\sigma+1}$ $\neq X$. Suppose $x \in X$ with $x \notin X^{\sigma}$. Since $X$ is saturated, there exist $y, z$ $\epsilon X$, separated in $X$, with $x \in S y \cap S z$. Hence $S y$ is not $X^{\sigma}$-distinguished, so that $y \notin X^{\sigma+1}$. This gives $X^{\sigma+1} \neq X$. Hence $h(X)$ must be a limit ordinal.

Theorem 5. Let $S$ be a monoid satisfying the d.c.c. on orbits, and let $X$ be a left $S$-operand. If $h(X)$ is finite, then $s l(X) \leq h(X)$. In fact, if $h(X)=n$, then $X_{n}=\varnothing$.

Proof. It suffices to show that if $X_{m} \neq \varnothing$ for a positive integer $m$, then $X^{m} \neq X$. Suppose now that $X_{m} \neq \varnothing$. Let $x_{m} \in X_{m}$. Then by Proposition 1 , there exist $x_{m-1}, y_{m-1} \in X_{m-1}$, separated in $X_{m-1}$, such that $x_{m} \in S x_{m-1} \cap S y_{m-1}$. Inductively, having found $x_{m-k}, y_{m-k} \in X_{m-k}$ for $0<k<m$, one finds $x_{m-k-1}, y_{m-k-1} \in X_{m-k-1}$, separated in $X_{m-k-1}$, such that $x_{m-k} \in S x_{m-k-1} \cap S y_{m-k-1}$. We do this for all $0<k \leq m$. Now $x_{m} \notin X^{0}$. Assuming inductively that $x_{m-k+1} \notin X^{k-1}$, one finds, since $S x_{m-k}$ and $S y_{m-k}$ are incomparable and $x_{m-k+1} \in S x_{m-k} \cap$ $S y_{m-k}$, that $x_{m-k} \notin X^{k}$. Hence one finds that $x_{0} \notin X^{m}$, so that $X^{m} \neq X$.

Now if $h(X)=n$ for some integer $n$, then $X^{n}=X$ so that $X_{n}=\varnothing$. Thus $s l(X) \leq n=h(X)$.

Corollary 6. Let $S$ and $X$ be as above; if $h(X)$ is finite, then the set of all maximal generalized orbits of $X$ forms an irredundant cover of $X$.

Proof. $X$ is the union of its maximal generalized orbits, and since $h(X)$ is finite, no maximal generalized orbit is contained in the union of the others.

Theorem 7. Let $S$ be a monoid satisfying the d.c.c. on orbits and let $X$ be a left $S$-operand. 
(i) The inequality of Theorem 5 can be strict. In fact, $h(X)-s l(X)$ can be made an arbitrary positive integer.

(ii) If $h(X)$ is infinite, then it can happen that $h(X)<s l(X)$.

Proof. (i) follows from Example 8 below, and (ii) follows from Example 9 below.

Example 8. Let $S=\left\{1, t, t^{2}, \cdots, t^{m-1}, t^{m}=t^{m+1}=t^{m+2}=\cdots\right\}$, with $m \geq 1$, be the monoid. Let $X=\left\{x_{0}, x_{1}, \cdots, x_{m}, y_{0}, y_{1}, \cdots, y_{m-1}\right\}$, and let $S$ act on $X$ by $t x_{i}=t y_{i}=x_{i+1}$ for $0 \leq i<m$ and $t x_{m}=x_{m}$. Then $X_{1}=$ $\left\{x_{1}, \cdots, x_{m}\right\}=S x_{1}$, and $X_{2}=\varnothing$ so that $s l(X)=2$. However, $X^{1}=\left\{x_{m}\right\}$, $X^{2}=\left\{x_{m-1}, y_{m-1}, x_{m}\right\}, X^{3}=\left\{x_{m-2}, y_{m-2}\right\} \cup X^{2}$, and, in general, $X^{k+1}=$ $\left\{x_{m-k}, y_{m-k}\right\} \cup X^{k}$ for $1 \leq k \leq m$. Hence $X^{m} \neq X$, but $X^{m+1}=X$, so that $h(X)=m+1$. Thus, if $k$ is a positive integer and $m=1+k$, then $h(X)-$ $s l(X)=k$.

Example 9. For the monoid take $N_{\min }=\{0,1,2, \ldots\} \cup\{I\}$, where $I$ is a two-sided identity and $a \cdot b=\min (a, b)$ otherwise. It is an easy direct computation (using the remark following Theorem 3.3 of [2]) to verify that $N_{\mathrm{m} \text { in }}$ satisfies the d.c.c. on orbits. Let $\omega$ denote the first infinite ordinal. Let

$$
\begin{array}{r}
X(\omega)=\left\{\left(x_{0}, x_{1}, \cdots, x_{n}, 0, \cdots\right): x_{i} \geq 0 \text { integers with } x_{i}=0\right. \\
\left.\forall i>x_{0}, \text { such that if } x_{n} \neq 0, \text { then } x_{k} \neq 0 \quad \forall 0 \leq k \leq n\right\} .
\end{array}
$$

For $k \geq 1$ an integer, let

$$
\begin{array}{r}
Y(k)=\left\{\left(k, x_{1}, \cdots, x_{k}, x_{k+1}, \cdots, x_{k+m}, 0, \cdots\right): x_{i} \geq 0 \text { integers } w i t h\right. \\
\left.x_{k+i}=0 \forall i>x_{k}, \text { such that if } x_{n} \neq 0, \text { then } x_{r} \neq 0 \forall 0 \leq r \leq n\right\} .
\end{array}
$$

Then let $X(\omega+k)=X(\omega) \cup Y(1) \cup \cdots \cup Y(k)$. (This union is formed with all sets considered as subsets of $N^{N}$, and can be pictured as follows: $X(\omega)$ is an infinite tree rooted at $(0,0,0, \ldots)$ and the $Y(j)$ are extra branches pasted onto $X(\omega)$.) The $X(\omega+k)$ for $k=0,1,2, \cdots$ become $N_{\text {min }}$ operands under the following action. Let $m \in N_{\mathrm{m} \text { in }}$ and $\left(x_{0}, \cdots, x_{n}, 0, \ldots\right)$ $\epsilon X(\omega+k)$ with $x_{n} \neq 0$; then

$$
\begin{gathered}
m \cdot\left(x_{0}, x_{1}, \cdots, x_{n}, 0, \cdots\right)=\left\{\begin{array}{l}
\left(x_{0}, x_{1}, \cdots, x_{n}, 0, \cdots\right) \text { if } x_{0}+\cdots+x_{n} \leq m, \\
\left(x_{0}, x_{1}, \cdots, x_{r-1}, x_{r}, 0, \cdots\right) \text { if for } r<n,
\end{array}\right. \\
x_{0}+\cdots+x_{r} \leq m<x_{0}+\cdots+x_{r}+x_{r+1}, \\
\quad \text { and } t=m-\left(x_{0}+\cdots+x_{r}\right) .
\end{gathered}
$$


Hence the sum of the coordinates of the new point is equal to $\min \left(m, x_{0}+x_{1}+\cdots+x_{n}\right)$. (This action may be visualized by thinking of the nodes along the direct path from $(0,0,0, \ldots)$ to the point in question of $X(\omega+k)$ as being renamed $0,1,2, \ldots$; then the path looks like an ideal in $N_{\min }$, and $N_{\min }$ acts as it would by multiplication on that ideal.) Note that $Y(k)$ as defined above is not a suboperand of $X(\omega+k)$.

For any integer $k \geq 1, h(X(\omega+k))=\omega$. To see this, first set $X=$ $X(\omega+k)$, then for $\alpha=\left(x_{0}, x_{1}, \cdots, x_{n}, 0, \ldots\right) \in X$, set $m(\alpha)=x_{0}+x_{1}+$ $\cdots+x_{n}$. Then show by induction that $\alpha \in X^{m(\alpha)}$ if $m(\alpha) \neq 0$. First observe that $\alpha \in X$ and $m(\alpha)=0$ if and only if $a=(0,0, \ldots)$, and that $m(\alpha)=1$ if and only if $\alpha=(1,0, \ldots)$, which generates an orbit which is comparable with every other orbit; hence, $(1,0,0, \ldots) \in X^{1}$. Now suppose that $a \in X^{m(\alpha)}$ for all $a$ with $0<m(\alpha) \leq p$. Let $\alpha=\left(x_{0}, \ldots, x_{n}, 0, \ldots\right)$ with $m(\alpha)=p+1$. For all $q \in N_{\mathrm{min}}, m(q \alpha)=\min (q, m(\alpha))$ so that either $q \alpha=\alpha$, or else $m(q \alpha) \leq p$, so that $q \alpha \in X^{p}$. Thus, if $\alpha$ is not already in $X^{p}$, then $N_{\mathrm{min}} \cdot a$ is an $X^{p}$-distinguished orbit of $X$ so that $a \in X^{p+1}$. This completes the induction showing that $h(X) \leq \omega$. One can show easily that $(m+1,0, \ldots) \notin X^{m}$ for all $m \geq 0$, so that $h(X) \geq \omega$. Hence $h(X)=\omega$.

Now one can show that $\operatorname{sl}(X(\omega+k))=\omega+k$ when $k \geq 2$. Let $X=$ $X(\omega+k)$. One observes first that if

$$
\alpha=\left(x_{0}, x_{1}, \cdots, x_{n}, 0, \cdots\right), \beta=\left(y_{0}, y_{1}, \cdots, y_{m}, 0, \cdots\right) \in X
$$

with $x_{n} \neq 0$ and $y_{m} \neq 0$, then $\alpha \in N_{\min } \cdot \beta$ if and only if $n \leq m, x_{i}=y_{i}$ for $i=0, \cdots, n-1$, and $x_{n} \leq y_{n}$; in this case $\alpha=m(\alpha) \cdot \beta$.

One next shows by induction that for all nonnegative integers $j$, $\left(x_{0}, \cdots, x_{n}, 0, \cdots\right) \in X_{j}$, with $x_{n} \neq 0$, if and only if (1) $k<x_{0}$ and $n \leq$ $\max \left(x_{0}-j, 0\right)$, or (2) $x_{0}=p \leq k$ and $n \leq p+\max \left(x_{p}-j, 0\right)$. (The two cases must be distinguished because of the effect of the $\left.Y(i), i=1, \ldots, k_{.}\right)$This statement is trivally true for $X_{0}=X$. Suppose now that it is true for $X_{j}$.

Let $\alpha=\left(x_{0}, x_{1}, \cdots, x_{n}, 0, \ldots\right)$ with $x_{n} \neq 0$.

Suppose first that $k<x_{0}$. Suppose $n \leq \max \left(x_{0}-j-1,0\right)$. We may assume that $x_{0} \geq j+1$. (If not, then $n=0$ and $\alpha \in N_{\min } \cdot \alpha^{\prime}$ where $\alpha^{\prime}=(j+1,0, \ldots)$ and where $\alpha^{\prime}$ satisfies the same condition as $\alpha$. If $\alpha^{\prime} \in X_{j+1}$, so is $\left.\alpha_{0}\right)$ Let $\beta=\left(x_{0}\right.$, $\left.x_{1}, \cdots, x_{n}+1,0, \ldots\right)$ and $\gamma=\left(x_{0}, \ldots, x_{n}, 1,0, \ldots\right)$. Since $x_{0} \geq n+1$, we have $\beta, \gamma \in X$. Then $\alpha, \beta, \gamma \in X_{j}$ by the inductive hypothesis, $\alpha \in\left(N_{\min } \cdot \beta\right) \cap$ $\left(N_{\min } \cdot \gamma\right)$, and $\beta$ and $\gamma$ are separated in $X$, hence in $X_{j}$. Thus $\alpha \in X_{j+1}$. 
On the other hand, if $\alpha=\left(x_{0}, \ldots, x_{n}, 0, \ldots\right) \in X_{j}$, with $x_{n} \neq 0$ and with $x_{0}>k$, then by inductive hypothesis $n \leq \max \left(x_{0}-j, 0\right)$. If $n=x_{0}-$ $j>0$, then one finds that if $a \in N_{\mathrm{m} \text { in }} \beta$ for some $\beta \in X_{j}$, it must be that $\beta=\left(x_{0}, x_{1}, \cdots, x_{n-1}, r, 0, \cdots\right)$ for some $r \geq x_{n}$. Thus if $\alpha \in N_{\min } \cdot \gamma$ for some $\gamma \in X_{j}$, either $\beta \in N_{\mathrm{min}} \cdot \gamma$ or $\gamma \in N_{\mathrm{min}} \cdot \beta$. Hence $\alpha$ lies in a unique maximal generalized orbit of $X_{j}$ so that $a \notin X_{j+1}$. This completes the induction for the case $x_{0}>k$.

Now suppose that $x_{0} \leq k$. Suppose $x_{0}=p$ and $n \leq p+\max \left(x_{p}-j-1,0\right)$. We may assume that $x_{p} \geq j+1$. (If not, then by an argument similar to that for the previous case, if $x_{p}<j+1$, then $n \leq p$. If $n=p$, we may replace $\alpha$ by $\alpha^{\prime}=\left(x_{0}, x_{1}, \cdots, x_{n-1}, j+1,0, \ldots\right)$; if $n<p$, we may replace $\alpha$ by $\alpha^{\prime}=\left(x_{0}, x_{1}, \cdots, x_{n}, x_{n+1}, \cdots, x_{p-1}, j+1,0, \cdots\right)$, where $x_{n}=$ $\left.x_{n+1}=\cdots=x_{p-1}.\right)$ Then as above, if $\beta=\left(x_{0}, \cdots, x_{n}+1,0, \ldots\right)$ and $y=\left(x_{0}, \cdots, x_{n}, 1,0, \cdots\right)$, then $\beta, \gamma \in X, \alpha, \beta, \gamma \in X_{j}$ by inductive hypothesis, $\alpha \in\left(N_{\min } \cdot \beta\right) \cap\left(N_{\min } \cdot \gamma\right)$, and $\beta$ and $\gamma$ are separated in $X_{j}$. Hence $a \in X_{j+1}$.

On the other hand, if $\alpha=\left(x_{0}, x_{1}, \ldots, x_{n}, 0, \ldots\right) \in X_{j}$, with $x_{n} \neq 0$ and $x_{0}=p \leq k$, then by the inductive hypothesis $n \leq p+\max \left(x_{p}-j, 0\right)$. If $n=p+x_{p}-j>p$, then, as above, one finds that if $\alpha \in N_{\mathrm{min}} \cdot \beta$ for $\beta \in X_{j}$, then $\beta=\left(x_{0}, \cdots, x_{n-1}, r, 0, \ldots\right)$ with $r \geq x_{n}$. Hence as before, $\alpha \notin X_{j+1}$. This completes the induction.

Now one finds that

$$
X_{\omega}=\{(u, 0, \cdots): k<u\} \cup\left\{\left(x_{0}, \cdots, x_{n}, 0, \cdots\right): x_{0} \leq k \text { and } n \leq x_{0}\right\} \subset X .
$$

To see this, first observe that if $u>k$, then $(u, 0, \ldots) \in X_{j}$ for all $j$ and $(u, 1,0, \ldots) \notin X_{u}$ by the above, so that $\left(x_{0}, x_{1}, \ldots, x_{n}, 0, \ldots\right) \in X_{\omega}$ with $k<x_{0}$ if and only if $n=0$. Likewise, if $x_{0}=p \leq k,\left(x_{0}, \cdots, x_{p}\right.$, $0, \cdots) \in X_{j}$ for all $j$ and $\left(x_{0}, \ldots, x_{p}, 1,0, \ldots\right) \notin X_{u}$, where $u=x_{p}$, so that $\left(x_{0}, \cdots, x_{n}, 0, \cdots\right) \in X_{\omega}$ with $x_{0} \leq k$ if and only if $n \leq x_{0}$.

Finally, one applies the induction applied to $X$ to $X \omega$ and finds that

$$
X_{\omega+k-1}=\{(u, 0, \cdots): 0 \leq u \leq k\} \cup\{(k, v, 0, \cdots): 1 \leq v\},
$$

which is a generalized orbit, so that $X_{\omega+k}=\varnothing$. Hence $s l(X)=\omega+k$. This shows that

$$
s l(X(\omega+k))=\omega+k>\omega=b(X(\omega+k)) \quad \text { if } k \geq 2,
$$

as desired. 


\section{REFERENCES}

1. A. H. Clifford and G. B. Preston, The algebraic theory of semigroups. Vol. 2, Math. Surveys, no. 7, Amer. Math. Soc., Providence, R.I., 1967. MR 36 \#1558.

2. W. R. Nico, A study of operands in terms of maximal generalized orbits, J. Algebra 30 (1974), 473-484.

DEPARTMENT OF MATHEMATICS, TULANE UNIVERSITY, NEW ORLEANS, LOUISIANA 70118 